Applied Physiology, Nutrition, and Metabolism

Canadian Science Publishing Physiologie appliquée, nutrition et métabolisme

\title{
Postexercise whole-body sweating increases during muscle metaboreceptor activation in young men
}

\begin{tabular}{|r|l|}
\hline Journal: & Applied Physiology, Nutrition, and Metabolism \\
\hline Manuscript ID & apnm-2017-0675.R1 \\
\hline Manuscript Type: & Rapid communication \\
\hline Complete List of Authors: & $\begin{array}{l}\text { Friesen, Brian; University of Ottawa, Human Kinetics } \\
\text { Poirier, Martin; University of Ottawa, School of Human Kinetics, Faculty of } \\
\text { Health Sciences } \\
\text { Lamarche, Dallon; University of Ottawa, Human Kinetics } \\
\text { D'Souza, Andrew; University of Ottawa, Human Kinetics } \\
\text { Kim, Jung-Hyun; Centers for Disease Control and Prevention, National } \\
\text { Institute for Occupational Safe } \\
\text { Notley, Sean; University of Ottawa, Faculty of Health Sciences } \\
\text { Kenny, Glen; University of Ottawa, }\end{array}$ \\
\hline Keyword: & exercise, heat loss, nonthermal, isometric, sweat rate \\
\hline $\begin{array}{r}\text { Is the invited manuscript for } \\
\text { consideration in a Special } \\
\text { Issue? : }\end{array}$ & N/A \\
\hline
\end{tabular}




\section{Postexercise whole-body sweating increases during muscle metaboreceptor activation in young men}

${ }^{1}$ Brian J. Friesen, ${ }^{2}$ Martin P. Poirier, ${ }^{3}$ Dallon T. Lamarche, ${ }^{4}$ Andrew W. D'Souza, ${ }^{5}$ Jung-Hyun Kim, ${ }^{6}$ Sean R. Notley and ${ }^{7}$ Glen P. Kenny

${ }^{1}$ Human and Environmental Physiological Research Unit, Faculty of Health Sciences, University of Ottawa, Ottawa, Ontario, Canada, K1N 6N5; bfrie051@uottawa.ca

${ }^{2}$ Human and Environmental Physiological Research Unit, Faculty of Health Sciences, University of Ottawa, Ottawa, Ontario, Canada, K1N 6N5; mpoir026@uottawa.ca

${ }^{3}$ Human and Environmental Physiological Research Unit, Faculty of Health Sciences, University of Ottawa, Ottawa, Ontario, Canada, K1N 6N5; dlama059@uottawa.ca

${ }^{4}$ Human and Environmental Physiological Research Unit, Faculty of Health Sciences, University of Ottawa, Ottawa, Ontario, Canada, K1N 6N5; adsou102@uottawa.ca

${ }^{5}$ Centers for Disease Control and Prevention, National Institute for Occupational Safety and Health, Pittsburgh, USA; inr3@cdc.gov

${ }^{6}$ Human and Environmental Physiological Research Unit, Faculty of Health Sciences, University of Ottawa, Ottawa, Ontario, Canada, K1N 6N5; snotley@uottawa.ca

${ }^{7}$ Human and Environmental Physiological Research Unit, Faculty of Health Sciences, University of Ottawa, Ottawa, Ontario, Canada, K1N 6N5; gkenny@uottawa.ca

\section{Corresponding author:}

Glen P. Kenny

University of Ottawa

125 University Ave.

Ottawa, ON, Canada, K1N 6N5

Phone: +1 6135625800 ext. 4282; Fax: +1 6135625497

E-mail: gkenny@uottawa.ca 


\section{ABSTRACT}

36 We assessed the effect of metaboreceptor activation on whole-body evaporative heat loss (WB-

37 EHL) in twelve men (24 \pm 4 years) in the early-to-late-stages of a 60 -min exercise recovery in the

38 heat. Metaboreceptor activation induced by 1-min isometric-handgrip (IHG) exercise followed

39 by 5 -min forearm ischemia to trap metabolites increased WB-EHL by $25-31 \%$ and $26-34 \%$

40 during the ischemic period relative to IHG-Only and Control (natural recovery only) respectively

41 throughout recovery. We show that metaboreceptor activation enhances WB-EHL in recovery.

42

43 Key words: exercise, heat loss, calorimetry, nonthermal, isometric, sweat rate.

44 


\section{INTRODUCTION}

An increasing number of studies demonstrate that nonthermal factors associated with the activation of sensory end organs of baroreceptors, osmoreceptors and metaboreceptors play an important role in the regulation of heat loss responses during heat stress (Kenny et al. 2010; Kondo et al. 2010). For instance, studies show that independent of thermal control, nonthermal factors can simultaneously act on the thermoregulatory system to modulate heat dissipation during the postexercise recovery period (Kenny and Journeay 2010; Kenny et al. 2016; Kenny and McGinn 2017). Upon cessation of exercise, the rapid decrease in metabolic heat liberation is paralleled by a decline in local and whole-body heat loss to pre-exercise resting levels despite sustained elevations in body temperatures (i.e. core and muscle) and therefore body heat storage (Kenny et al. 2008; Kenny and Journeay 2010). This response, which has been shown to last for up to 120 minutes, has been primarily attributed to the overriding influences of nonthermal factors on postexercise thermoregulatory function (Kenny et al. 2008; Kenny and Journeay 2010).

.

Until recently, the nonthermal mediated postexercise disturbance in thermal homeostasis was largely ascribed to a baroreflex-mediated response associated with postexercise hypotension (Kenny and Journeay 2010). However, recent findings reveal that other nonthermal factors associated with metaboreceptor activation may be involved (Kenny and McGinn 2017, McGinn et al. 2014; Paull et al. 2015). The accumulation of metabolites within the active skeletal muscle has been shown to stimulate group III and IV afferent neurons which in turn evoke a reflex increase in muscle sympathetic nerve activity, known as the muscle metaboreflex (Rowell and O'Leary 1990). The activation of metaboreceptors have been shown to modulate heat loss 
responses during both passive heat stress and exercise (Crandall et al. 1998; Shibasaki et al. 2001; Boushel 2010) with recent studies extending their influence to the postexercise period (McGinn et al. 2014; Paull et al. 2015). These latter studies demonstrate that the influence of baroreceptors may be limited to the control of local skin blood flow whereas metaboreceptors, and not baroreceptors, may largely influence the regulation of local sweating (McGinn et al. 2014; Paull et al. 2015); an important avenue of heat dissipation during heat stress. Specifically, metaboreceptor activation, induced by a brief isometric handgrip (IHG) exercise followed by forearm ischemia to trap metabolites, was shown to delay the rapid reduction in local sweating occurring in the early stages of recovery ( $\leq 20 \mathrm{~min})(\mathrm{McGinn}$ et al. 2014; Paull et al. 2015) while increasing local sweating during the mid-to-late stages of recovery (30- to 60-min of recovery)(Paull et al. 2015) relative a natural recovery (no IHG or ischemia). Despite regional variations in absolute local sweat rate, a consistent response was observed across different body regions (i.e., chest, forearm and upper back)(Paull et al. 2015) during the activation of metaboreceptors. Taken together, these findings indicate that the metaboreceptor-mediated modulation of local sweating may have an important effect on whole-body sweating. In this context, it is possible that changes in metaboreceptor activity in the postexercise period may play an important role in the observed disruption of thermal homeostasis.

Thus, in the present study, we examined the effect of metaboreceptor activation on whole-body sweating by assessing whole-body evaporative heat loss (WB-EHL) during the early- (15 min), mid- (30 min) and late- (45 min) stages of recovery following a 45-min moderate intensity exercise bout in the heat $\left(35^{\circ} \mathrm{C}, 20 \%\right.$ relative humidity). To evaluate this response, we used direct calorimetry to precisely measure WB-EHL (Kenny et al. 2017). We evaluated the hypothesis that following dynamic exercise, WB-EHL would increase in response 
91 to the activation of metaboreceptors induced by a period of post-IHG ischemia throughout the

92 early-to-late stages of the 60-min recovery period relative to both IHG exercise only and a

93 natural recovery.

\section{METHODS}

\section{Ethical approval}

The experimental protocol was approved by the University of Ottawa Health Sciences and Science Research Ethics Board and agrees with the Declaration of Helsinki. Written and

99 informed consent was obtained from all participants prior to their participation in the study.

\section{Participants}

101

Twelve healthy habitually active young males (age: $24 \pm 4$ years; height: $1.7 \pm 0.7 \mathrm{~m}$; body

102 mass: $76.0 \pm 8.1 \mathrm{~kg}$; body surface area: $1.90 \pm 0.11 \mathrm{~m}^{2}$; peak oxygen consumption $\left(\dot{\mathrm{V}}_{2 \text { peak }}\right)$ : 49.4 $\pm 7.7 \mathrm{mLO}_{2} / \mathrm{kg} / \mathrm{min}$; body fat percentage: $16.9 \pm 5.4 \%$ ) participated in this study.

\section{Experimental Design}

Participants completed one preliminary and three experimental sessions each separated

107 by at least 48 hours. For all sessions, participants refrained from exercise, alcohol or caffeine 24

108 hours prior to experimentation. During the preliminary session, the participant's physical 109 characteristics (height, weight, and body composition) was assessed. Additionally, aerobic

110 fitness (defined by $\dot{\mathrm{V}}_{2 \text { peak }}$ ) was determined through an incremental cycling exercise protocol.

111 Participants performed two 5-sec maximal voluntary contractions (MVC) to establish the 
112 relative intensity (determined using the higher of the two values) for the isometric handgrip 113 (IHG) exercise. The detailed procedures are reported elsewhere (McGinn et al. 2014; Paull et al. 114 2015). For each experimental session, following instrumentation, participants rested inside the 115 direct air calorimeter regulated at $35^{\circ} \mathrm{C}(20 \%$ relative humidity) for a 20 -min resting period (Preexercise resting) followed by a 45-min bout of cycling at a fixed rate of metabolic heat

117 liberation of $400 \mathrm{~W}$ (to maintain a constant thermal drive for sweating between conditions) and a 118 60-minute recovery. During recovery, they performed either: 1) a 1-min IHG exercise at $60 \%$ of 119 their predetermined MVC (IHG-Only), 2) a 1-min IHG at 60\% of MVC followed by a 5-min 120 forearm ischemia (IHG+OCC) or 3) a natural recovery with no IHG or OCC (Control). 121 Forearm occlusion was achieved by inflating a blood pressure cuff to supra-systolic levels which 122 was then deflated rapidly for a 5-min recovery period. The protocols were repeated in the early 123 (15 $\mathrm{min})$, mid (30 $\mathrm{min})$ and late $(45 \mathrm{~min})$ stages of the 60 -min recovery data collection period. 124 During the period of muscle ischemia, it is thought that the accumulation of metabolites within 125 the muscle triggers chemosensitive afferents (group III and IV afferents) and reflexively raises 126 arterial blood pressure which is used to confirm the activation of metaboreceptors (Rowell and 127 O'Leary 1990).

\section{Instrumentation}

The modified Snellen direct air calorimeter was used to perform continuous measurements of whole-body evaporative (WB-EHL) and dry heat loss (WB-DHL), and metabolic heat liberation was calculated by indirect calorimetry (Kenny et al. 2017). Esophageal

132 temperature was measured using a thermocouple probe (Mallinckrodt Medical Inc., St-Louis, 133 MO, USA) estimated to be in the region bounded by the left ventricle and aorta. Skin 134 temperature was measured using $0.3-\mathrm{mm}$ diameter T-type (copper/constantan) thermocouples 
135 integrated into heat flow sensors (Concept Engineering, Old Saybrook, CT, USA) affixed to the 136 chest, biceps, thigh, and calf temperatures (Concept Engineering, Old Saybrook, CT, USA).

137 Mean skin temperature was calculated using the following weightings: chest, 30\%; bicep, 30\%;

138 thigh, 20; and calf, 20\% (Ramanathan 1964). Calorimetry and temperature data were recorded 139 continuously on a personal computer with LabVIEW software (version 7.0; National 140 Instruments, Austin, TX, USA). Mean arterial blood pressure (MAP) (Finapres Medical Systems, 141 Amsterdam, The Netherlands) was measured continuously. Isometric handgrip exercise was 142 performed using a Smedley Hand Dynamometer (Model 19117, Stoelting Co, Wood Dale, IL, 143 USA).

\section{Data analysis}

Pre-exercise resting and end-exercise values were averaged over the last 5-min. For the postexercise recovery, values for each of the three recovery periods were assessed based on the

147 four time stages for the metaboreceptor activation protocol as follows: 1) 1-min period preceding 148 the IHG (Pre-IHG), 2) last $15 \mathrm{sec}$ of the IHG exercise (IHG), 3) the final 15 sec of the 149 postexercise forearm occlusion (OCC), and 4) final $15 \mathrm{sec}$ of the post-OCC recovery period 150 (REC). Body heat storage was calculated as the temporal summation of metabolic heat liberation 151 and heat loss within the 5-min occlusion phase during each recovery stage.

\section{Statistical analysis}

A one-way analysis of variance (ANOVA) with the non-repeated factor of condition

155 (Control, IHG-Only, IHG+OCC) was conducted for all dependent variables for pre-exercise 156 resting, end-exercise as well as for the change in body heat storage across conditions. When a 157 main effect of condition was measured, post-hoc analysis was conducted using paired sample t- 
158 tests corrected for multiple comparisons using the Bonferroni procedure. During recovery, a two159 way repeated measures ANOVA was performed to compare the dependent variables at each 160 stage (Pre-IHG, IHG, OCC, REC) as well as between the three experimental conditions (3 levels: 161 Control, IHG-Only, IHG+OCC) for each recovery period. When a significant main effect or 162 interaction was observed, post hoc comparisons were carried out using paired-sample t-tests 163 corrected for multiple comparisons using the Bonferroni procedure. Results are presented at 164 means \pm SD. All statistical analyses performed using IBM SPSS Statistics v24.0 (SPSS Inc., 165 Chicago, IL, USA).

\section{RESULTS}

\section{Pre-exercise resting and End-exercise}

Pre-exercise resting and end-exercise responses for body temperatures (esophageal: $36.9 \pm 0.3^{\circ} \mathrm{C}, 37.3 \pm 0.4^{\circ} \mathrm{C}$; mean skin: $\left.34.4 \pm 0.5^{\circ} \mathrm{C}, 35.0 \pm 0.5^{\circ} \mathrm{C}\right)$, MAP $(92 \pm 5 \mathrm{mmHg}, 94 \pm 7$ $\mathrm{mmHg})$, metabolic heat liberation $(108 \pm 18 \mathrm{~W}, 390 \pm 31 \mathrm{~W})$, and heat loss (WB-EHL: $86 \pm 38 \mathrm{~W}$,

$172373 \pm 51 \mathrm{~W}$; WB-DHL: $-10 \pm 18 \mathrm{~W},-25 \pm 19 \mathrm{~W}$ ) respectively were similar between conditions (all $173 \mathrm{P}>0.05)$.

174 Postexercise recovery

176 phases in all recovery stages (all P>0.05), averaging $113 \pm 17,110 \pm 19$ and $108 \pm 15 \mathrm{~W}$ (early), $177106 \pm 13,108 \pm 18$ and $104 \pm 12 \mathrm{~W}$ (mid) and $110 \pm 16,114 \pm 17$ and $107 \pm 14 \mathrm{~W}$ (late) across 178 conditions, respectively. Metabolic heat liberation was also similar between conditions during 179 IHG exercise during the early-stage ( $\mathrm{P}=0.11$; mean across conditions: $123 \pm 18 \mathrm{~W}$ ), yet was 
180 elevated in the mid- and late-stages relative to Control (107 \pm 15 and $101 \pm 11 \mathrm{~W}$, respectively) in 181 the IHG+OCC condition ( $124 \pm 21$ and $124 \pm 18 \mathrm{~W}$, respectively; $\mathrm{P}=0.02$ and $\mathrm{P}=0.01)$ and in the 182 IHG-only condition in the mid- $(122 \pm 12 \mathrm{~W} ; \mathrm{P}=0.04)$, but not the late-stage $(114 \pm 18 \mathrm{~W} ; \mathrm{P}=0.26)$. 183 WB-DHL did not differ between conditions or phases in each recovery stage (all P>0.05), 184 averaging $-5 \pm 17,-2 \pm 19$ and $1 \pm 18 \mathrm{~W}$ across phases during the early-, mid- and late-stages, 185 respectively. For all recovery stages, WB-EHL increased during the IHG exercise for the IHG186 only and IHG+OCC conditions relative to Control (all $\mathrm{P}<0.05$ ), and remained elevated during the 187 OCC phase for the IHG+OCC condition relative to the Control and IHG-only conditions (all $188 \mathrm{P}<0.05$; Figure 1). Heat stored during OCC was reduced during the early-, mid- and late-stages 189 190 191 192 phases (all $\mathrm{P}>0.05$ ), but increased during IHG exercise for IHG-only and IHG+OCC conditions 194 relative to the Control condition (all $\mathrm{P}<0.05$ ) and remained elevated during OCC in the 195 IHG+OCC condition relative to the IHG-only and Control conditions (all $\mathrm{P}<0.05$; Figure 1). Esophageal and mean skin temperatures remained elevated above baseline but similar between 197 conditions during the early- (esophageal: $37.1 \pm 0.3^{\circ} \mathrm{C}$, mean skin: $34.7 \pm 0.5^{\circ} \mathrm{C}$ ), mid198 (esophageal: $37.1 \pm 0.3^{\circ} \mathrm{C}$, mean skin: $34.6 \pm 0.5^{\circ} \mathrm{C}$ ) and late-stages (esophageal: $37.1 \pm 0.3^{\circ} \mathrm{C}$, mean 199 skin: $34.6 \pm 0.4^{\circ} \mathrm{C}$; all $\left.\mathrm{P}>0.05\right)$. 


\section{DISCUSSION}

We show for the first time that the activation of muscle metaboreceptors augments whole-body evaporative heat loss (and therefore whole-body sweating) and that the level of influence (as defined by the relative increase in WB-EHL of $\sim 45 \mathrm{~W}$ ) remains constant throughout the early-to-late stages of recovery despite a progressive decay in body temperatures. Specifically, we showed that metaboreceptor activation induced by 1-min isometric-handgrip (IHG) exercise followed by 5-min forearm ischemia to trap metabolites increased WB-EHL by $25-31 \%$ and $26-34 \%$ during the ischemic period relative to IHG-Only and Control (no-IHG or ischemia, natural recovery) respectively throughout recovery. Moreover, despite the relatively short duration of the period of stimulation (i.e., 5-min ischemia), the metaboreceptor-mediated increase in evaporative heat loss was associated with a concomitant reduction in body heat storage. Taken together, our findings demonstrate that the metaboreceptor modulation of wholebody sweating is an important factor mediating the postexercise disturbance in thermal homeostasis.

Previous studies have demonstrated that the activation of metaboreceptors play an important role in the regulation of local forearm sweating (McGinn et al. 2014; Paull et al. 2015) as well as back and upper chest sweating (Paull et al. 2015) during the postexercise period. This response was shown to be independent of any changes in baroreceptor loading status that occurs during the occlusion period (McGinn et al. 2014). While previous studies showed that the activation of metaboreceptors preserved but did not augment local sweating in the early stages of recovery (i.e., $\leq 20-\mathrm{min}$ ) (McGinn et al. 2014; Paull et al. 2015), we observed a consistent increase in whole-body sweating throughout the early-to-late stages of recovery. This disparity may in part be due differences in the level of hyperthermia achieved during the exercise. In 
226 previous studies, the level of hyperthermia prior to the start of the metaboreceptor activation

227 protocol was greater (as defined by a greater rise in core temperature of $\sim 0.3-0.6^{\circ} \mathrm{C}$; equivalent

228 to $\left.\geq 37.4^{\circ} \mathrm{C}\right)$ relative to that recorded in the present study $\left(\sim 37.0^{\circ} \mathrm{C}\right)$. In keeping with this

229 observation, previous work showed that thermal control predominates over nonthermal factors at 230 core temperatures above $\sim 37.4^{\circ} \mathrm{C}$ (Gagnon et al. 2008). Differences in the duration of the period 231 of ischemia may also contribute to this response. While prior studies employed a 2 min period of 232 ischemia, we used an extended period of ischemia $(5 \mathrm{~min})$ to ensure that a metaboreceptor233 mediated response, if any, would be captured in our calorimetric measurement of whole-body 234 sweating. It remains to be determined if the increase in whole-body sweating would have been 235 observed with a shorter period of ischemia.

In summary, we show that metaboreceptor activation enhances whole-body WB-EHL 237 and therefore whole-body sweating, throughout the early-to-late stages of the postexercise 238 recovery period in the heat. This was associated with a 56, 88 and $115 \%$ greater amount of heat 239 stored during the natural recovery (i.e. Control) relative to recovery with the activation of 240 metaboreceptors (i.e. IHG+OCC) performed in the early-, mid- and late-stages respectively. Our 241 findings demonstrate that independent of thermal control (i.e. changes in skin and/or core 242 temperatures), changes in nonthermal metaboreceptor activity during the postexercise period 243 play an important role in the observed disruption in whole-body thermal homeostasis. In the 244 context of exercise or work performed in the heat, our findings demonstrate that nonthermal 245 factors can influence the rate of heat dissipation and therefore the restoration of thermoregulation 246 after exercise in the heat. 


\section{ACKNOWLEDGEMENTS}

250

251

252

253

254

255

256

257

258

259

260

261

262

263

264

265

266
We thank all the participants who volunteered for the present study. This research was supported by the Natural Sciences and Engineering Research Council (RGPIN-298159-2009, RGPIN-2014-06313, held by Dr. Glen P. Kenny). Disclaimer: The findings and conclusions in this article are those of the authors and do not necessarily represent the views of the National institute for Occupational Safety and Health.

\section{DISCLOSURES}

No conflict of interest, financial or otherwise, are declared by the author(s). The results of the study are presented clearly, honestly, and without fabrication, falsification, or inappropriate data manipulation.

\section{AUTHOR CONTRIBUTIONS}

G.P.K. and B.J.F. conceptualized and designed the research; B.J.F., M.P.P., D.T.L. and A.W.D. performed experiments; B.J.F. and M.P.P. analyzed the data; all authors interpreted the results of experiments; G.P.K. drafted the manuscript; all authors edited and revised the manuscript and approved final version of manuscript. 


\section{REFERENCES}

268 Boushel, R. 2010. Muscle metaboreflex control of the circulation during exercise. Acta Physiol.

269 (Oxf.) 199: 367-383. doi: 10.1111/j.1748-1716.2010.02133.x. PMID: 20353495.

270 Crandall, C., Stephens, D., and Johnson, J. 1998. Muscle metaboreceptor modulation of

271 cutaneous active vasodilation. Med. Sci. Sports Exerc. 30: 490-496. PMID: 9565928.

272 Gagnon, D., Jay, O., Reardon, F.D., Journeay, W.S., and Kenny, G.P. 2008. Hyperthermia

273 modifies the nonthermal contribution to postexercise heat loss responses. Med. Sci. Sports Exerc.

274 40: 513-522. doi: 10.1249/MSS.0b013e31815eb7b8. PMID: 18379215

275 Kenny, G.P. and Journeay, W.S. 2010. Human thermoregulation: separating thermal and

276 nonthermal effects on heat loss. Front. Biosci. 15: 259-290. PMID: 20036820.

277 Kenny, G.P. and McGinn, R. 2017. Restoration of thermoregulation after exercise. J. Appl.

278 Physiol. 122:933-944. doi: 10.1152/japplphysiol.00517.2016. PMID: 27881668.

279 Kenny, G.P., Notley, S.R., and Gagnon, D. 2017. Direct calorimetry: a brief historical review of 280 its use in the study of human metabolism and thermoregulation. Eur. J. Appl. Physiol. 117: 1765-

281 1785. doi: 10.1007/s00421-017-3670-5. PMID: 28689303.

282 Kenny, G.P., Webb, P., Ducharme, M.B., Reardon, F.D., and Jay, O. 2008. Calorimetric

283 measurement of postexercise net heat Loss and residual body heat storage. Med. Sci. Sports

284 Exerc. 40: 1629-1636.

285 Kondo, N., Nishiyasu, T., Inoue, Y., and Koga, S. 2010. Non-thermal modification of heat-loss

286 responses during exercise in humans. Eur. J. Appl. Physiol. 110: 447-458. doi:

287 10.1249/MSS.0b013e31817751cb. PMID: 18685528. 
288 McGinn, R., Swift, B., Binder, K., Gagnon, D., and Kenny, G.P. 2014. Do metaboreceptors alter 289 heat loss responses following dynamic exercise? Am. J. Physiol. Regul. Integr. Comp. Physiol. 290 306: R82-89. PMID: 24226030.

291 Paull, G., Dervis, S., McGinn, R., Haqani, B., Flouris, A.D., Kondo, N., and Kenny, G.P. 2015.

292 Muscle metaboreceptors modulate postexercise sweating, but not cutaneous blood flow, 293 independent of baroreceptor loading status. Am. J. Physiol. Regul. Integr. Comp. Physiol. 309:

294 R1415-1424. doi: 10.1152/ajpregu.00287.2015. PMID: 26377560.

295 Ramanathan, N.L. 1964. A New Weighting System for Mean Surface Temperature of the Human 296 Body. J. Appl. Physiol. 19: 531-533. PMID: 14173555

297 Rowell, L.B. and O'Leary, S. 1990. Reflex control of circulation during exercise: chemoreflexes 298 and mechanoreflexes. J. Appl. Physiol. 69: 407-418. PMID: 2228848.

299 Shibasaki, M., Kondo, N., and Crandall, C.G. 2001. Evidence for metaboreceptor stimulation of 300 sweating in normothermic and heat-stressed humans. J. Physiol. 534: 605-611. PMID: 11454976. 


\section{FIGURE CAPTION}

305 Figure 1. Whole-body evaporative heat loss (W) (Panel A) and mean arterial pressure (mmHg)

306 (Panel B) during Pre-isometric hand grip exercise (Pre-IHG, 1 min prior to IHG), last 15 sec of 307 isometric handgrip exercise (IHG), last $15 \mathrm{sec}$ of forearm occlusion (OCC) and last $15 \mathrm{sec}$ of 308 recovery from forearm occlusion (REC, 5 min after the end of OCC) during Control, IHG-Only 309 and IHG+OCC conditions. Measurements were performed in the early- (15 min), mid- (30 min) 310 and late- (45 min) stages of postexercise recovery. *Significantly different between IHG+OCC 311 and Control. †Significantly different between IHG-Only and Control. $¥$ Significantly different 312 between IHG+OCC and IHG-Only. Significance level accepted at $\mathrm{P} \leq 0.05$. Values are presented 313 as mean $\pm \mathrm{SD}$. 
$15 \mathrm{~min}$

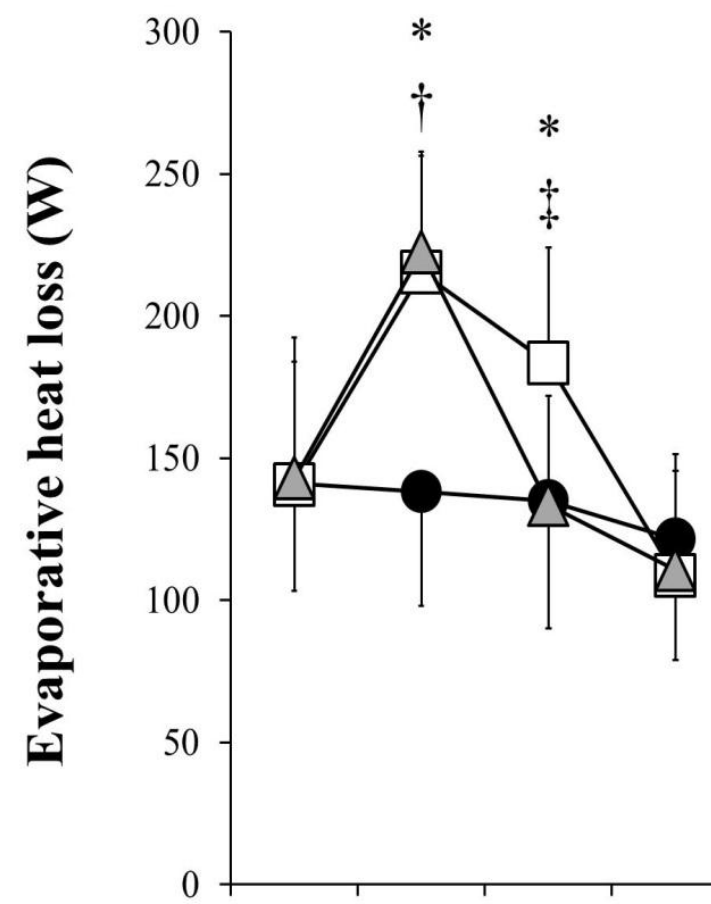

$30 \min$

$45 \min$

B

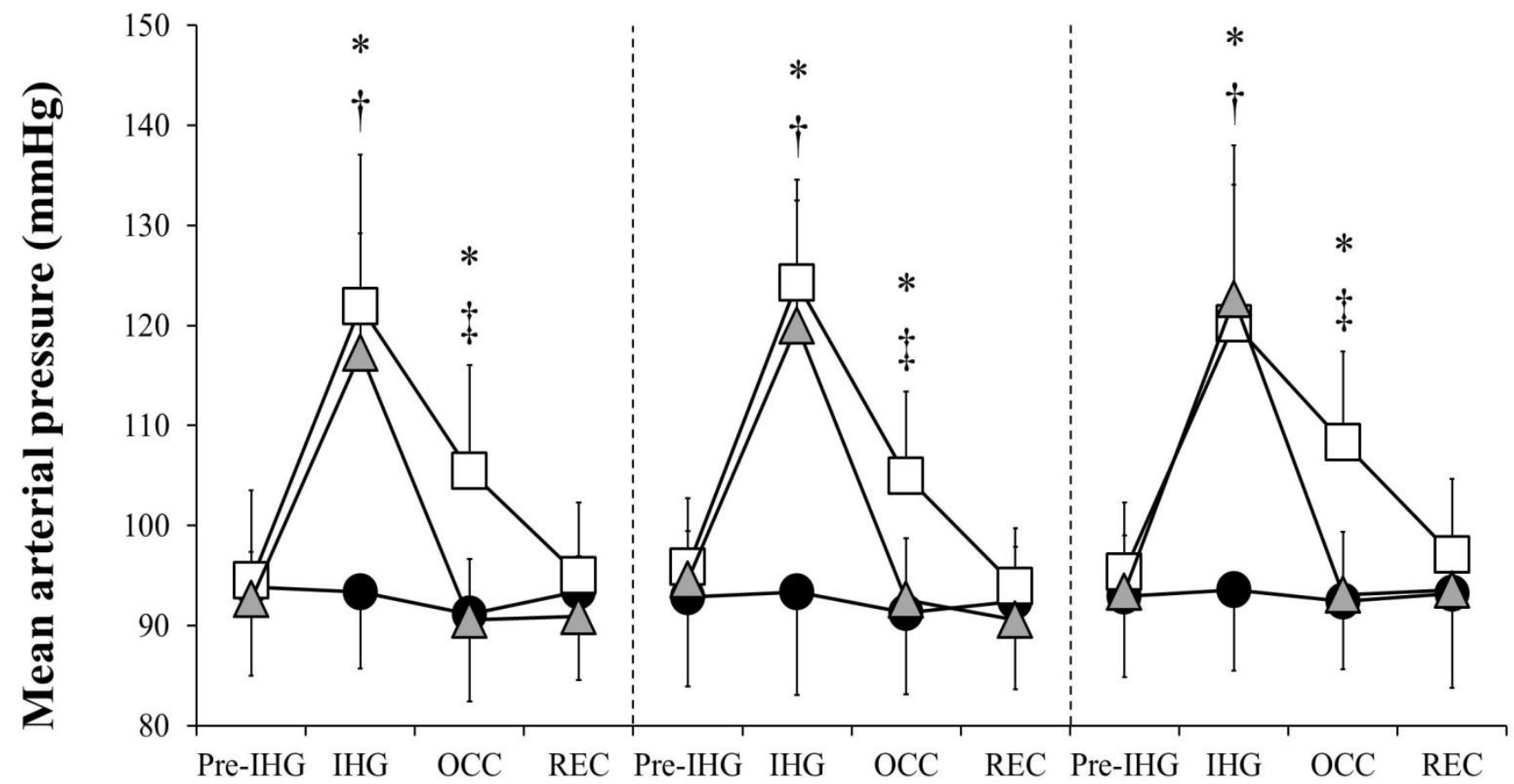

